\title{
Índices de estacionalidad de los precios medios recibidos por los productores de manzanas chilenas
}

\author{
Germán Lobos Andrade(1) y Tristán Muñoz Ibáñez(2)
}

(1)Universidad de Talca, Facultad de Ciencias Empresariales, Casilla 721, Talca, Chile. E-mail: globos@utalca.cl (2)Arrocera Tucapel S.A.I.C., Casilla 14, Retiro, Chile. E-mail: curimaqui@tie.cl

\begin{abstract}
Resumen - El objetivo de este trabajo fue analizar el comportamiento de los precios medios FOB (franco a bordo) de manzanas frescas chilenas. A partir de este análisis, se estimaron los precios medios recibidos por los productores. Se estimaron patrones de estacionalidad ajustada de los precios recibidos por los productores de manzanas chilenas, a través del método del promedio geométrico móvil. Para las estimaciones se consideraron los precios FOB promedio mensuales desde enero de 1990 a septiembre de 2004. Los resultados mostraron: una baja estacionalidad de precios; una estabilidad de precios para junio y desde septiembre a diciembre; un valor máximo en julio. Los precios más bajos ocurrieron en abril y mayo. La principal conclusión, desde el punto de vista económico, sugiere que la rentabilidad de los huertos de manzanos podría ser incrementada, a través de un mejoramiento en el proceso de planificación de la producción.
\end{abstract}

Términos para indexación: estacionalidad ajustada, promedio móvil, estabilidad de precios, rentabilidad.

\section{Seasonal indices for mean prices received by Chilean apple farmers}

\begin{abstract}
The purpose of this paper is to analyze the behavior of FOB (free on board) average prices for the Chilean fresh apples. Based on this analysis, the average prices received by farmers were estimated. The patterns of seasonally adjusted price fluctuations, received by Chilean apple farmers, were estimated through average mobile geometric method. Estimates considered monthly average FOB prices from January 1990 up to September 2004. Results showed: a low seasonal price pattern; price stability for June and from September to December; the peak value in July. The lowest prices occurred in April and May. From an economic point of view, the main conclusion suggests that apple orchard profitability could be increased by an improved yield planning.

Index terms: seasonally adjusted, mobile average, price stability, profitability.
\end{abstract}

\section{Introducción}

Durante la temporada 2003/2004, la producción mundial de manzanas alcanzó a 43 millones de toneladas, lo que representa una caída del $2 \%$ con respecto a la última temporada y del $10 \%$ con respecto a la cifra record de 48 millones de toneladas del periodo 2000/2001. La mayor reducción se observó en algunos de los principales países del Hemisferio Norte, tales como China, Federación Rusa e Italia, mientras que la producción aumentó en EE.UU., Turquía y Polonia. En el caso de China, principal productor mundial (43\%), la caída en la producción se explica por la tendencia decreciente en los precios domésticos e internacionales de las manzanas, lo que se ha traducido en una reducción en estas plantaciones y en una diversificación hacia variedades frutales más rentables. Entre los países del Hemisferio
Sur, los cuales aportan un 10\% de la producción mundial de manzanas, se observó un aumento en Chile, Nueva Zelanda y Sudáfrica, mientras que una declinación en Brasil, Australia y Argentina (USDA, 2004).

En Chile, actualmente, la superficie plantada con manzanos se estima en 35.020 ha, de la cual un $81 \%$ corresponde a manzano rojo y el $19 \%$ restante a manzano verde; la superficie de manzanos representa alrededor de un $18 \%$ del total de las plantaciones de frutales mayores, concentradas básicamente en la VI y VII Región. Las principales variedades de manzanas comercializadas en los mercados internacionales son Royal Gala, Granny Smith y Richard Delicius; en menor medida, se comercializan Fuji, Red Starking, Braebum y las demás variedades. Según cifras de ODEPA (2004), el volumen de exportaciones de manzanas frescas chilenas pasó de 314 a 596 millones de kilogramos entre 1990 y 2003. 
En general, la alta volatilidad en los precios agrícolas ha sido atribuida: a las bajas elasticidades precio-propio e ingreso de la demanda por productos agrícolas; a la inestabilidad de la producción agrícola, derivada de efectos externos no controlables por los productores (Sekhar, 2003a, 2003b); y a la naturaleza del proceso de producción agrícola, donde las decisiones deben tomarse en un contexto de riesgo (González et al., 2002a, 2002b), respecto de las condiciones que prevalecerán en los mercados de destino (Starleaf, 1982).

Lee (1994), Duval \& Biere (2002), Agbola (2003) y Stewart et al. (2003) sugieren que las frutas frescas son un bien normal y que la demanda es inelástica con respecto al precio-propio. En un estudio sobre demanda por importaciones de manzanas frescas chilenas en la Unión Europea, Cerda et al. (2004) obtuvieron valores de 0,913 para la elasticidad ingreso de la demanda y de -0,368 para la elasticidad precio-propio, considerando a éste último como el precio relativo de las manzanas chilenas, con respecto al precio de las manzanas exportadas por China.

Con datos de series de tiempo mensuales del periodo 1995 a 2001, Morgado et al. (2004) calcularon índices de estacionalidad de los precios medios recibidos por los productores de piñas en Rio de Janeiro, Brasil, con base en una media geométrica móvil centralizada en 12 meses. Los autores concluyeron que los precios medios presentan cierta estabilidad de enero a octubre, mientras que los valores más bajos se observaron en los meses de noviembre y diciembre, periodo en que se concentra la producción.

Troncoso y Lobos (2004) utilizaron datos de series de tiempo mensuales, del periodo 1993 a 2002, y analizaron la estabilidad de los precios y márgenes de comercialización para una canasta de frutas y hortalizas en Chile. Cada serie de tiempo fue descompuesta en cuatro componentes (tendencia, estacionalidad, ciclicidad y estocástica), de acuerdo al modelo propuesto por Mendenhall \& Reinmuth (1981) y Maddala (1996). Según los autores, la componente estacional es la más relevante en todas las series analizadas.

Por otra parte, en un estudio que incluyó 425 encuestas a nivel nacional, Domínguez et al. (2003) concluyeron que, según los productores frutícolas chilenos, los principales aspectos que deberían regularse en la relación entre productores y exportadoras es la información sobre los precios de venta en los mercados de destino (20,2\%) y la transparencia en la información (19,1\%).
Además, las principales razones para explicar una positiva relación con la exportadora fueron la buena relación profesional $(16,8 \%)$, buenos precios $(11,4 \%)$ y buena asesoría técnica (10,8\%). El objetivo de este trabajo fue analizar el comportamiento de los precios medios FOB de manzanas frescas chilenas.

\section{Material y Métodos}

En este trabajo se analizó el comportamiento de los precios medios de las manzanas frescas chilenas mediante el cálculo de índices de estacionalidad. Con base en los precios medios FOB, medidos en US\$ $\mathrm{kg}^{-1}$, obtenidos de los registros de ODEPA (2004), se realizó una estimación de los precios medios recibidos por los productores de manzanas, una vez descontadas las comisiones y tarifas cobradas por las empresas exportadoras. Para las estimaciones, se utilizaron datos mensuales del periodo enero de 1990 a septiembre de 2004. Los precios medios FOB mensuales nominales fueron convertidos a valores reales de septiembre de 2004, utilizándose como deflactor el Índice de Precios de Importación (MPI) para frutas y sus preparaciones, incluso jugos frescos (base septiembre $2000=100$ ), reportado por la Oficina de Estadísticas Laborales (BLS, 2004) de EE.UU. (Cuadro 1).

Para estimar los precios medios mensuales recibidos por los productores, se utilizó el procedimiento indirecto de cálculo de su valor; éste consiste en descontar a los precios medios FOB mensuales los valores pagados por los productores a las empresas exportadoras, los cuales incluyen la comisión de exportación y las tarifas por servicios; de esta manera, los precios así estimados pueden ser asimilados a los precios medios recibidos por los productores. Para ello se usó la siguiente expresión:

$$
\mathrm{P}_{\mathrm{ij}}^{\mathrm{PRO}}=\mathrm{P}_{\mathrm{ij}}^{\mathrm{FOB}}-\mathrm{cP}_{\mathrm{ij}}^{\mathrm{FOB}}-\sum_{\mathrm{t}=1}^{\mathrm{n}} \mathrm{T}_{\mathrm{t}} \text {, }
$$

donde $\mathrm{P}^{\mathrm{PRO}}$ es el precio medio de las manzanas recibido por los productores (US\$ kg-1); los subíndices $\mathrm{i}, \mathrm{j}$ corresponden al año y mes, respectivamente; c es la comisión de exportación (la cual fue estimada en 8\%); T son las tarifas (US\$ kg-1) cobradas por las exportadoras; $y$ el subíndice t se refiere al tipo de tarifa. Entre estas últimas se incluyen los costos de frío, proceso (o packing), ducha bins (o aplicación del antioxidante Difenilamina, DPA), uso de bins, control de calidad, certificación del Servicio Agrícola y Ganadero (SAG), 
convenios de control de calidad con la Asociación de Exportadores de Chile, materiales de embalaje, fletes desde la planta al puerto, embarque y estiba. La información sobre las tarifas fue obtenida de diferentes empresas exportadoras; para efectos de este trabajo se usaron valores aproximados. Para comparar la variabilidad de los precios medios de cada una de las series, se calculó el coeficiente de variación (CV), el cual permitió conocer, en porcentaje, la variabilidad de los precios medios con respecto a su promedio. Los precios medios estimados recibidos por los productores se presentan en el Cuadro 2.

El precio medio es igual al producto de cuatro componentes (Hanke \& Reitsch, 1997; Morgado et al., 2004; Troncoso \& Lobos, 2004): 1) un factor T que incluye la tendencia de largo plazo de una serie de tiempo; 2) un factor $\mathrm{C}$ que representa las variaciones cíclicas alrededor de la tendencia, y que en algunos casos reflejan las oscilaciones suaves que contiene una serie de tiempo debido a la actividad económica, y cuya periodicidad fluctúa entre 2 y 5 años; 3) un factor E que representa las variaciones estacionales, las cuales corresponden a fluctuaciones interanuales, que se repiten regularmente año a año; y 4) un factor I que representa la componente irregular (oscilaciones no sistemáticas), la cual mide las variaciones aleatorias de una serie de tiempo, después de eliminar las otras componentes. Lo anterior se puede expresar como $\mathrm{P}_{\mathrm{ij}}=\mathrm{P}_{\mathrm{t}}=\mathrm{T}_{\mathrm{t}} \mathrm{C}_{\mathrm{t}} \mathrm{E}_{\mathrm{t}} \mathrm{I}_{\mathrm{t}}$, donde los subíndices $\mathrm{i}, \mathrm{j}$ corresponden al año y mes, respectivamente, y el subíndice t se refiere al valor en el periodo t, considerándose el carácter discreto de la serie de datos.

En este trabajo se adoptó la metodología de promedios geométricos móviles, descrita por Hanke \& Reitsch (1997), para determinar los patrones de variación estacional y un intervalo de dispersión de índices estacionales de los precios medios, recibidos por los productores de manzanas frescas chilenas, utilizándose datos de series de tiempo mensuales del periodo enero de 1990 a septiembre de 2004.

Se calculó el total móvil de 12 meses, de enero a diciembre de 1990, para los datos del primer año, el cual fue centrado el $1^{\circ}$ de julio de 1990. En el siguiente total móvil de 12 meses, se excluyó el precio medio de enero de 1990 y se incluyó enero de 1991, el cual fue centrado el 1 o de agosto de 1990. Siguiendo este procedimiento, en el último total móvil de 12 meses, se consideró los precios medios de octubre de 2003 a septiembre de 2004, y fue centrado el 1o de abril de 2004. Se calculó el total móvil de 2 años, con datos de 24 meses, sumándose los totales móviles centrados el 10 de cada mes. El primer valor de la serie fue centrado el 15 de julio de 1990; siguiendo este procedimiento, el último valor fue centrado el 15 de marzo de 2004. Se dividió el total móvil de 2 años entre 24 para obtenerse el promedio de 12 meses, centrados el 1 o de cada mes.

Se calculó el índice estacional (IE) para cada mes, dividiéndose el precio medio real recibido por los productores entre el promedio centrado de 12 meses;

Cuadro 1. Precios medios FOB de manzanas frescas chilenas, moneda de septiembre de 2004 (US\$ kg-1).

\begin{tabular}{|c|c|c|c|c|c|c|c|c|c|c|c|c|c|c|c|}
\hline Mes & 1990 & 1991 & 1992 & 1993 & 1994 & 1995 & 1996 & 1997 & 1998 & 1999 & 2000 & 2001 & 2002 & 2003 & 2004 \\
\hline Ene. & 0,33 & 0,67 & 0,57 & 0,55 & 0,44 & 0,44 & 0,67 & 2,06 & 0,44 & 0,41 & 0,35 & 0,33 & 0,34 & 0,44 & 0,43 \\
\hline Feb. & 0,34 & 0,37 & 0,34 & 0,45 & 0,39 & 0,68 & 0,86 & 0,50 & 0,59 & 0,55 & 0,45 & 0,43 & 0,45 & 0,50 & 0,53 \\
\hline Mar. & 0,29 & 0,32 & 0,33 & 0,33 & 0,35 & 0,36 & 0,40 & 0,43 & 0,54 & 0,48 & 0,45 & 0,41 & 0,50 & 0,48 & 0,53 \\
\hline Abr. & 0,28 & 0,33 & 0,39 & 0,34 & 0,36 & 0,36 & 0,36 & 0,41 & 0,45 & 0,41 & 0,44 & 0,38 & 0,42 & 0,46 & 0,48 \\
\hline Mayo & 0,28 & 0,34 & 0,38 & 0,38 & 0,37 & 0,38 & 0,38 & 0,41 & 0,51 & 0,39 & 0,46 & 0,38 & 0,43 & 0,52 & 0,50 \\
\hline Jun. & 0,36 & 0,44 & 0,46 & 0,38 & 0,42 & 0,40 & 0,42 & 0,45 & 0,52 & 0,44 & 0,55 & 0,44 & 0,46 & 0,56 & 0,51 \\
\hline Jul. & 0,64 & 0,52 & 0,71 & 0,37 & 0,50 & 0,46 & 0,52 & 0,55 & 0,54 & 0,52 & 0,55 & 0,47 & 0,47 & 0,55 & 0,45 \\
\hline Ago. & 0,45 & 0,54 & 0,59 & 0,43 & 0,48 & 0,44 & 0,52 & 0,50 & 0,48 & 0,46 & 0,48 & 0,40 & 0,43 & 0,51 & 0,49 \\
\hline Sep. & 0,40 & 0,51 & 0,54 & 0,43 & 0,52 & 0,46 & 0,48 & 0,49 & 0,43 & 0,40 & 0,44 & 0,33 & 0,38 & 0,41 & 0,45 \\
\hline Oct. & 0,39 & 0,44 & 0,70 & 0,45 & 0,50 & 0,47 & 0,48 & 0,50 & 0,43 & 0,37 & 0,45 & 0,35 & 0,36 & 0,42 & - \\
\hline Nov. & 0,41 & 0,48 & 0,46 & 0,41 & 0,76 & 0,47 & 0,47 & 0,61 & 0,39 & 0,38 & 0,46 & 0,35 & 0,39 & 0,45 & - \\
\hline Dic. & 0,44 & 0,43 & 0,53 & 0,43 & 0,43 & 0,57 & 1,95 & 0,48 & 0,42 & 0,37 & 0,41 & 0,35 & 0,36 & 0,41 & - \\
\hline Promedio & 0,38 & 0,45 & 0,50 & 0,41 & 0,46 & 0,46 & 0,63 & 0,62 & 0,48 & 0,43 & 0,46 & 0,39 & 0,42 & 0,48 & $\overline{0,49}$ \\
\hline Desvest & 0,10 & 0,10 & 0,13 & 0,06 & 0,11 & 0,09 & 0,44 & 0,46 & 0,06 & 0,06 & 0,05 & 0,05 & 0,05 & 0,05 & 0,04 \\
\hline $\mathrm{CV}(\%)$ & 26 & 23 & 26 & 14 & 24 & 20 & 70 & 74 & 13 & 14 & 12 & 12 & 12 & 11 & 7 \\
\hline
\end{tabular}

Fuente: Elaboración propia, en base a datos de la Oficina de Estudios y Políticas Agrarias (ODEPA, 2004). 
este resultado fue multiplicado por 100, para convertir la razón en un número índice. Se obtuvo un IE para un total de 165 meses, desde julio de 1990 a marzo de 2004, con 13 IE para los meses de abril, mayo y junio, y 14 para cada uno de los restantes meses (Cuadro 3).

Se obtuvo la media mensual modificada (MMM), mediante el promedio simple de los IE, eliminándose el valor máximo y mínimo de cada mes, con el objetivo de eliminar cualquier distorsión de los valores extremos.

Se calculó el índice estacional ajustado (IEA) dividiéndose 1.200 entre la suma de las MMM, y multiplicándose el resultado obtenido por el correspondiente índice estacional mensual. Se calculó el índice de irregularidad ajustado (IIA), siguiéndose el mismo procedimiento de cálculo del IEA. Para ello fue necesario estimar el valor de tendencia pronosticado y el índice cíclico (IC) para cada mes; este último valor, en conjunto con el promedio móvil centrado en 12 meses, permitió obtener el índice cíclico irregular (ICI). El índice de irregularidad se calculó, dividiéndose el ICI entre el IC. Finalmente, se estableció un intervalo de dispersión de dos índices estacionales (límites superiores e inferiores), el cual fue obtenido multiplicándose y dividiéndose el IEA por el IIA (Cuadro 4).

Cuadro 2. Precios medios estimados a productor, en base a precios medios FOB de manzanas frescas chilenas, moneda de septiembre de $2004\left(\mathrm{US} \$ \mathrm{~kg}^{-1}\right)$.

\begin{tabular}{|c|c|c|c|c|c|c|c|c|c|c|c|c|c|c|c|}
\hline Mes & 1990 & 1991 & 1992 & 1993 & 1994 & 1995 & 1996 & 1997 & 1998 & 1999 & 2000 & 2001 & 2002 & 2003 & 2004 \\
\hline Ene. & 0,20 & 0,50 & 0,41 & 0,40 & 0,29 & 0,29 & 0,51 & 1,78 & 0,30 & 0,27 & 0,21 & 0,19 & 0,20 & 0,29 & 0,29 \\
\hline Feb. & 0,20 & 0,23 & 0,21 & 0,30 & 0,25 & 0,52 & 0,68 & 0,34 & 0,43 & 0,39 & 0,31 & 0,29 & 0,30 & 0,35 & 0,38 \\
\hline Mar. & 0,15 & 0,18 & 0,19 & 0,19 & 0,21 & 0,22 & 0,26 & 0,28 & 0,39 & 0,33 & 0,30 & 0,27 & 0,34 & 0,33 & 0,38 \\
\hline Abr. & 0,14 & 0,19 & 0,25 & 0,20 & 0,22 & 0,22 & 0,22 & 0,27 & 0,31 & 0,27 & 0,29 & 0,24 & 0,28 & 0,32 & 0,33 \\
\hline Mayo & 0,15 & 0,20 & 0,24 & 0,23 & 0,23 & 0,24 & 0,24 & 0,27 & 0,36 & 0,25 & 0,31 & 0,24 & 0,29 & 0,37 & 0,35 \\
\hline Jun. & 0,22 & 0,29 & 0,31 & 0,24 & 0,27 & 0,26 & 0,28 & 0,30 & 0,36 & 0,30 & 0,39 & 0,29 & 0,32 & 0,40 & 0,36 \\
\hline Jul. & 0,47 & 0,37 & 0,54 & 0,23 & 0,35 & 0,31 & 0,37 & 0,40 & 0,39 & 0,37 & 0,40 & 0,32 & 0,33 & 0,39 & 0,30 \\
\hline Ago. & 0,31 & 0,38 & 0,43 & 0,29 & 0,33 & 0,30 & 0,37 & 0,35 & 0,33 & 0,32 & 0,33 & 0,26 & 0,29 & 0,36 & 0,34 \\
\hline Sep. & 0,26 & 0,35 & 0,38 & 0,28 & 0,37 & 0,31 & 0,33 & 0,34 & 0,28 & 0,25 & 0,30 & 0,19 & 0,23 & 0,27 & 0,30 \\
\hline Oct. & 0,25 & 0,29 & 0,53 & 0,30 & 0,35 & 0,32 & 0,33 & 0,35 & 0,28 & 0,23 & 0,30 & 0,21 & 0,22 & 0,28 & - \\
\hline Nov. & 0,26 & 0,33 & 0,32 & 0,26 & 0,59 & 0,32 & 0,32 & 0,45 & 0,25 & 0,24 & 0,31 & 0,21 & 0,25 & 0,30 & - \\
\hline Dic. & 0,29 & 0,29 & 0,38 & 0,29 & 0,28 & 0,41 & 1,68 & 0,33 & 0,27 & 0,23 & 0,27 & 0,21 & 0,22 & 0,27 & - \\
\hline Promedio & 0,24 & 0,30 & 0,35 & 0,27 & 0,31 & 0,31 & 0,46 & 0,46 & 0,33 & 0,29 & 0,31 & 0,24 & 0,27 & 0,33 & 0,34 \\
\hline Desvest & 0,09 & 0,09 & 0,12 & 0,05 & 0,10 & 0,08 & 0,40 & 0,42 & 0,06 & 0,05 & 0,05 & 0,04 & 0,05 & 0,05 & 0,03 \\
\hline CV (\%) & 38 & 31 & 34 & 20 & 33 & 27 & 87 & 93 & 17 & 19 & 16 & 18 & 18 & 14 & 10 \\
\hline
\end{tabular}

Cuadro 3. Índices estacionales de precios medios recibidos por los productores de manzanas frescas chilenas.

\begin{tabular}{|c|c|c|c|c|c|c|c|c|c|c|c|c|c|c|c|}
\hline Mes & 1990 & 1991 & 1992 & 1993 & 1994 & 1995 & 1996 & 1997 & 1998 & 1999 & 2000 & 2001 & 2002 & 2003 & 2004 \\
\hline Ene. & - & 178,3 & 132,7 & 118,9 & 111,0 & 87,6 & 146,1 & 320,9 & 81,9 & 89,2 & 73,4 & 69,0 & 75,7 & 96,9 & 88,1 \\
\hline Feb. & - & 80,0 & 64,9 & & & & 192,2 & & & & & & 116,0 & 113,3 & 117,3 \\
\hline Mar. & - & 63,9 & 58,9 & 2,7 & 75,9 & 66,8 & 72,4 & 50,6 & 108,9 & 111,3 & 103,0 & 99,5 & 129,9 & 107,0 & 116,9 \\
\hline Abr. & - & & & & & & & & & & & & & & - \\
\hline Mayo & - & 67,9 & 70,4 & 4,5 & 76,4 & 75,9 & 66,4 & 48,0 & 104,0 & 86,5 & 102,4 & 94,1 & 106,5 & 114,4 & - \\
\hline Jun. & - & 96,6 & 91,2 & & 87,8 & & & & & 103,2 & 127,8 & 119,6 & 116,4 & 123,7 & \\
\hline Jul. & 185,9 & 124,1 & 154,7 & 86,3 & 111,3 & 97,2 & 70,6 & 101,2 & 118,4 & 128,7 & 128,0 & 133,2 & 118,3 & 120,4 & - \\
\hline Ago. & 113,8 & 130,9 & 122,1 & 112,3 & 101,5 & & 65,7 & 104,8 & 101,5 & 113,7 & 108,0 & 104,4 & 102,3 & 109,3 & - \\
\hline Sep. & 94,1 & 121,2 & 107,6 & 110,2 & 110,8 & 91,2 & 60,6 & 98,8 & & 92,9 & 96,7 & 77,3 & 82,8 & 81,4 & \\
\hline Oct. & 91,8 & 98,3 & 151,2 & 117,1 & 104,2 & 92,4 & 61,2 & 100,5 & 88,7 & 84,1 & 99,7 & 82,8 & 77,4 & 83,0 & \\
\hline Nov. & 94,9 & 112,0 & 90,0 & 102,0 & 175,9 & 92,4 & 58,6 & 125,7 & 80,0 & 85,3 & 104,4 & 83,1 & 85,4 & 91,0 & - \\
\hline Dic. & 103,2 & 95,3 & 107,9 & 110,4 & 84,5 & 119,4 & 304,0 & 91,3 & 90,4 & 81,5 & 92,8 & 80,6 & 73,5 & 81,0 & - \\
\hline
\end{tabular}




\section{Resultados y Discusión}

La comparación de los precios medios estimados a productor y los precios medios FOB (Cuadros 1 y 2) sugiere que las empresas exportadoras transfieren a los productores un porcentaje de variabilidad mayor que las que ellas enfrentan en los mercados internacionales de manzanas frescas chilenas; lo cual se comprueba al observar el CV anual, estimado para cada serie de precios. Lo anterior se explica por la fórmula de cálculo de los precios medios a productor, la cual incorpora una componente variable (comisión, expresada como porcentaje del precio medio FOB) y una componente fija (tarifas, expresada en US\$ $\mathrm{kg}^{-1}$ ).

La evolución de los precios medios estimados a productor, para las series original (Cuadro 3) y desestacionalizada, se muestran en la Figura 1. El análisis de la componente estacional ajustada, de los precios medios estimados a productor (Cuadro 4), indica que dichos precios son más altos que el promedio anual, en los meses de enero, febrero, y de junio a agosto. Durante todos los otros meses, los precios medios fueron inferiores al promedio anual. Los mayores precios medios se observaron durante los meses de julio y fueron superiores en un $23,1 \%$ al promedio anual. Por otra parte, los menores precios medios se observaron durante los meses de abril y fueron inferiores en un $18,4 \%$ al promedio anual.

El análisis gráfico de variación estacional y de límites superior e inferior de los precios medios (Cuadro 4) muestra que en el mes de junio y de septiembre a diciembre ocurre cierta estabilidad en dichos precios, mientras que la mayor variabilidad se observó en el mes de julio, con un IEA de 123,1 y límites de 152,43 (superior) y de 99,41 (inferior).

Los precios medios más bajos, observados durante los meses de abril y mayo, coinciden con el periodo de los mayores volúmenes exportados, lo cual coincide con la temporada de cosecha y mayor producción en el país (Cuadro 4). Por otra parte, los precios medios más altos, durante los meses de junio a agosto, son consistentes con las épocas de inicio de la temporada de producción en los países del Hemisferio Norte. Cabe destacar que los índices de estacionalidad de los precios medios, recibidos por los productores, estimados por Morgado et al. (2004), también mostraron valores más bajos, durante los meses en que se concentra la producción.

Los resultados obtenidos en este trabajo contribuyen a mejorar el conocimiento del funcionamiento de los mercados y a conocer el comportamiento de los precios, tanto para las empresas exportadoras como para los productores, tal como lo sugiere el trabajo de Domínguez et al. (2003). Además, permiten tomar mejores decisiones de producción y optimizar el proceso de cosecha, de manera de disminuir el riesgo asociado a los precios (González et al., 2002a, 2002b) y a las condiciones que prevalecerán en los mercados de destino, al momento de la comercialización (Starleaf, 1982). La alta volatilidad observada en los precios

Cuadro 4. Índices estacionales y límites (superior e inferior) de precios medios recibidos por los productores de manzanas frescas chilenas, de 1990 a 2004.

\begin{tabular}{|c|c|c|c|c|c|c|}
\hline \multirow[t]{2}{*}{ Mes } & \multirow{2}{*}{$\begin{array}{c}\text { Volumen de } \\
\text { exportaciones } \\
\text { (millones de kg) }\end{array}$} & \multirow{2}{*}{$\begin{array}{l}\text { Media mensual } \\
\text { modificada } \\
(\mathrm{MMM})\end{array}$} & \multirow{2}{*}{$\begin{array}{l}\text { Índice estacional } \\
\text { ajustado (IEA) }\end{array}$} & \multirow{2}{*}{$\begin{array}{c}\text { Índice de } \\
\text { irregularidad } \\
\text { ajustado (IIA) }\end{array}$} & \multicolumn{2}{|c|}{ Límites } \\
\hline & & & & & Superior & Inferior \\
\hline Ene. & 8,93 & 106,66 & 108,44 & 94,14 & 115,19 & 102,09 \\
\hline Feb. & 99,42 & 108,03 & 109,84 & 90,96 & 120,76 & 99,91 \\
\hline Mar. & 982,93 & 95,20 & 96,80 & 114,12 & 110,47 & 84,82 \\
\hline Abr. & $1.882,77$ & 80,30 & 81,65 & 119,65 & 97,69 & 68,24 \\
\hline Мауо & $1.415,98$ & 84,99 & 86,41 & 113,19 & 97,81 & 76,34 \\
\hline Jun. & 895,17 & 99,09 & 100,74 & 97,28 & 103,56 & 98,00 \\
\hline Jul. & 644,42 & 121,07 & 123,10 & 80,76 & 152,43 & 99,41 \\
\hline Ago. & 387,42 & 106,92 & 108,71 & 88,02 & 123,50 & 95,69 \\
\hline Sep. & 260,49 & 94,27 & 95,85 & 100,55 & 96,37 & 95,33 \\
\hline Oct. & 196,99 & 93,33 & 94,89 & 101,54 & 96,35 & 93,46 \\
\hline Nov. & 109,98 & 95,52 & 97,12 & 99,57 & 97,53 & 96,70 \\
\hline Dic. & 42,50 & 94,86 & 96,44 & 100,22 & 96,66 & 96,23 \\
\hline
\end{tabular}




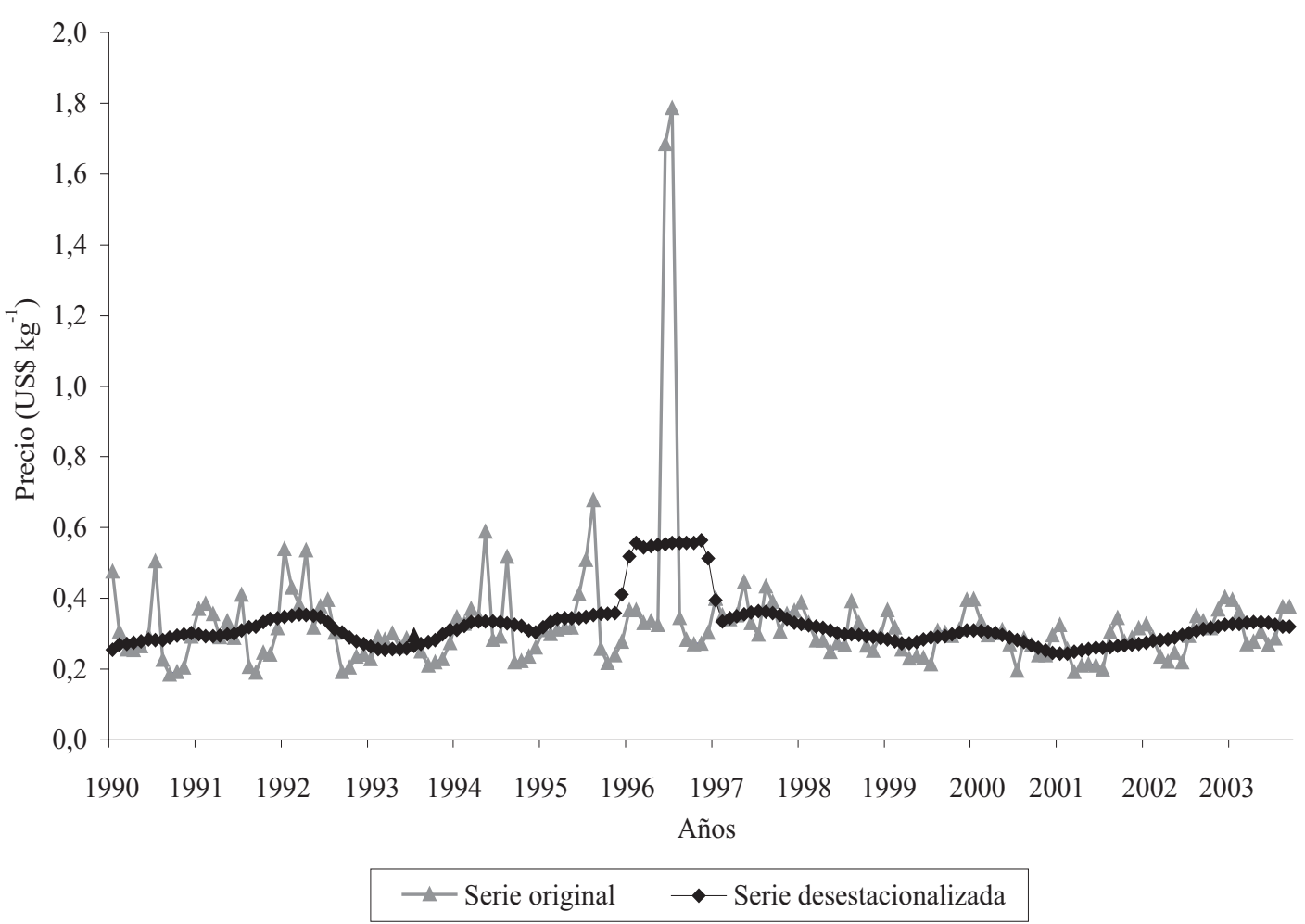

Figura 1. Serie original y desestacionalizada de precios medios estimados, recibidos por los productores de manzanas frescas chilenas, en el mes de julio, de 1990 a 2004.

internacionales de las manzanas frescas chilenas, la que es transmitida en una mayor proporción a los productores, es consistente con la clasificación de las manzanas frescas como bienes normales e inelásticos al precio-propio (Lee, 1994; Duval \& Biere, 2002; Agbola, 2003; Stewart et al., 2003; Cerda et al., 2004). Lo anterior se traduce en que los cambios en el ingreso y los cambios de precios afectan, menos que proporcionalmente, la demanda y la cantidad demandada de manzanas frescas, respectivamente.

\section{Conclusiones}

1. La variabilidad en los precios medios FOB de manzanas frescas chilenas no sólo afecta a las empresas exportadoras, sino que afecta en mayor medida a los productores, ya que las primeras transfieren a los segundos un porcentaje más alto de dicha variabilidad.

2. Considerándose los índices de estacionalidad y la variabilidad de los precios medios estimados a nivel productor, adquiere especial relevancia la labor desarrollada por los profesionales y extensionistas que atienden a los productores, en el sentido de contribuir a que éstos puedan mejorar el proceso de planificación de la producción, flexibilizando la época de cosecha.

\section{Referencias}

AGBOLA, F.W. Estimation of food demand patterns in South Africa based on a survey of households. Journal of Agricultural and Applied Economics, v.35, p.663-670, 2003.

BLS. Bureau of Labor Statistics. [Home page]. Disponible en: <http://www.bls.gov/mxp/home.htm>. Leído el: 19 aug. 2004.

CERDA, A.; LOBOS, G.; KUFFERATH, E.; SÁNCHEZ, F. Elasticidades de demanda por manzanas chilenas en el mercado de la Unión Europea: una estimación econométrica. Agricultura Técnica, v.64, p.399-408, 2004.

DOMÍNGUEZ, J.I.; SOLER, C.; ROJAS, F. Visión de productores y exportadores sobre aspectos clave para el desarrollo de la fruticultura chilena. Santiago, Chile: Pontificia Universidad Católica de Chile, 2003. 28p. Disponible en: <http://www.faif.puc.cl/ biblioteca/framebiblioteca.htm>. Leído el: 19 ago. 2004.

DUVAL, Y.; BIERE, A. Product diffusion and demand for new food products. Agribusiness, v.18, p.23-36, 2002.

GONZÁLEZ, J.; FRANCISCO, E.; FOSTER, W. Nivel y variabilidad del beneficio económico de rotaciones para la precordillera andina de la región del Bío-Bío. Agricultura Técnica, v.62, p.439449, 2002b. 
GONZÁLEZ, J.; FRANCISCO, E.; FOSTER, W. Selección de portfolios de rotaciones culturales económicamente óptimos para la precordillera andina de la VIII Región. Agricultura Técnica, v.62, p.583-595, 2002a.

HANKE, J.E.; REITSCH, A.G. Estadística para negocios. 2.ed. Madrid, España: McGraw-Hill, 1997. 961p.

LEE, J.Y. Fresh fruit consumption in Japan. Agribusiness, v.10, p.513-520, 1994.

MADDALA, G.S. Introducción a la econometría. 2.ed. México: Prentice-Hall, 1996. 715p.

MENDENHALL, W.; REINMUTH, J.E. Estadística para administración y economía. 3.ed. México: Iberoamérica, 1981. 707p.

MORGADO, I.F.; AQUINO, C.N.; TERRA, D.C. Economic aspects of the pineapple culture: seasonally of prices in Rio de Janeiro State. Revista Brasileira de Fruticultura, v.26, p.44-47, 2004.

ODEPA. Oficina de Estudios y Políticas Agrarias. [Home page]. Disponible en: <http://www.odepa.cl.htm>. Leído el: 5 ago. 2004.
SEKHAR, C.S.C. Price formation in world wheat markets implications for policy. Journal of Policy Modeling, v.25, p.85106, 2003a.

SEKHAR, C.S.C. Volatility of agricultural prices - an analysis of major international and domestic markets. Econpapers, 2003b. (Working paper, 103) 78p. Disponible en: <http:// www.econpapers.repec.org/paper/indicrier/103.htm>. Leído el: ago. 2004.

STARLEAF, D.R. Macroeconomic policies and their impact upon farm sector. American Journal of Agricultural Economics, v.64, p.854-860, 1982.

STEWART, H.; BLISARD, N.; JOLLIFE, D. Do income constraints inhibit spending on fruits and vegetables among low-income households? Journal of Agricultural and Resource Economics, v.28, p.465-480, 2003.

TRONCOSO, C.; LOBOS, G. Márgenes de comercialización y concentración industrial en el mercado de frutas y hortalizas en Chile. Agroalimentaria, v.18, p.75-86, 2004.

USDA. United States Department of Agriculture. [FAS online]. Disponible en: <http://www.fas.usda.gov/>. Leído el: 10 nov. 2004. 